\title{
Paraoxonase 1 polymorphisms and survival
}

\author{
L Christiansen $^{*, 1,2}$, L Bathum ${ }^{1,2}$, H Frederiksen $^{1}$ and K Christensen ${ }^{1}$ \\ ${ }^{1}$ Epidemiology, Institute of Public Health, University of Southern Denmark, Odense, Denmark; ${ }^{2}$ Department of Clinical \\ Biochemistry and Clinical Genetics, Odense University Hospital, Denmark
}

The antioxidant enzyme paraoxonase 1 (PON1) has previously been suggested to confer protection against coronary heart disease (CHD), one of the main causes of death in the Western world. Two coding polymorphisms, $55 \mathrm{M} / \mathrm{L}$ and $192 \mathrm{Q} / \mathrm{R}$, and a promoter variant, $-107 \mathrm{C} / \mathrm{T}$, has been extensively studied with respect to susceptibility to CHD. In this study, we have investigated the impact of these three

polymorphisms on mortality using a sample of 1932 Danish individuals aged 47-93 years, previously used in gene-longevity studies. A cross-sectional study comparing the genotype distribution of the three polymorphisms separately as well as the haplotype distribution in different age groups did not reveal any difference. However, a longitudinal follow-up study on survival in the same sample indicated that 192RR homozygotes have a poorer survival compared to $Q Q$ homozygotes (hazard rate: $1.38, P=0.04$ ). We hereafter used an independent sample of 541 Danish individuals from the oldest cohort and confirmed the initial findings (hazard rate: $1.38, P=0.09$ ). In both samples, the effect was most pronounced in women. Using self-reported data on ischemic heart disease to evaluate the impact of the PON 192Q/R polymorphism on susceptibility to CHD, we found only a nonsignificant trend of 192RR homozygosity in women being a risk factor. Our results thus indicates that PON1 192RR homozygosity is associated with increased mortality in women in the second half of life and that this increased mortality is possibly related to CHD severity and survival after CHD rather than susceptibility to development of CHD.

European Journal of Human Genetics (2004) 12, 843-847. doi:10.1038/sj.ejhg.5201235

Published online 7 July 2004

Keywords: paraoxonase, polymorphisms; mortality

\section{Introduction}

The antioxidant enzyme paraoxonase 1 (PON1) is found associated with high-density lipoproteins (HDLs) in plasma. Although the physiological substrate remains unknown, one suggested function of PON1 is the elimination of accumulated oxidized lipids in lipoproteins. ${ }^{1}$ Thus, PON1 has been suggested to be responsible for the ability of HDL to prevent oxidative modification of low-density lipoprotein (LDL), thereby allegedly conferring protection against coronary heart disease (CHD), one of the main causes of death in the Western world..$^{1-4}$

*Correspondence: Dr L Christiansen, Department of Clinical Biochemistry and Clinical Genetics, Odense University Hospital, Sdr. Boulevard 29, Odense C 5000, Denmark. Tel: +45 6541 2843; Fax: + 456541 1911; E-mail: Ichristiansen@health.sdu.dk

Received 8 March 2004; revised 22 April 2004; accepted 28 April 2004
Two polymorphisms, both resulting in amino-acid substitutions, L55M and Q192R, have been described within the coding region of the PON1 gene. ${ }^{5}$ In addition, Leviev et $a l^{6}$ identified three common polymorphisms in the promoter region of the PON1 gene, and suggested that all three to some extent affected the PON1 level. ${ }^{6}$ Investigating the influence of the two coding sequence polymorphisms on the efficacy of HDL's protection of LDL lipid oxidation, Mackness et $\mathrm{al}^{7}$ demonstrated that individuals with the PON1 192QQ/55MM genotype was most effective in protecting LDL against lipid oxidation, whereas PON1 192RR/55LL homozygotes was least effective. An examination of the relevance of the identified PON1 promoter polymorphisms have revealed that promoterrelated variations in PON1 concentration and activity was almost exclusively attributable to the $\mathrm{C} / \mathrm{T}$ polymorphic site at position -107 , with the $-107 \mathrm{C}$ allele being the high 
expresser allele. ${ }^{8}$ A recent meta-analysis of 43 studies of the 192Q/R, 55L/M and $-107 \mathrm{C} / \mathrm{T}$ polymorphisms involving 11212 CHD cases and 12786 controls suggested that there is no significant association with either $55 \mathrm{~L} / \mathrm{M}$ or $-107 \mathrm{C} / \mathrm{T}$ and $\mathrm{CHD}$, and only a weak overall association between 192Q/R and CHD. ${ }^{9}$

Few studies, however, have investigated the impact of PON1 polymorphisms on survival. Heijmans et $a l^{10}$ found no significant effect of the Q192R and M55L polymorphisms on mortality risk in 666 subjects age $85+$ years in a 10-year follow-up study, whereas Bonafé et al ${ }^{11}$ reported an increase in the frequency of $55 \mathrm{M} / 192 \mathrm{R}$ carriers in 308 centenarians as compared to 579 younger individuals. In this study, we examined the association between the $-107 \mathrm{C} / \mathrm{T}$, M55L and Q192R polymorphisms in a sample of Danish subjects aged 47-93 years drawn among participants of three populations-based studies. We used the sample both in a cross-sectional design and in a longitudinal follow-up study 5 years in average. All three polymorphism were investigated in a total of 1932 individuals and to confirm positive findings in the initial cohorts, a further 541 subjects 93 years of age were examined.

\section{Materials and methods Subjects}

The subjects included in this study were drawn from three major population-based surveys; the Danish 1905 Cohort, which includes all Danes born in $1905 ;^{12}$ the Longitudinal Study of Ageing Danish Twins (LSADT), including all Danish twins age 70 years or older ${ }^{13,14}$ the Study of Middle-aged Danish Twins (MADT), including Danish twins born between 1931 and 1952. ${ }^{14,15}$ Initially, we genotyped a total of 1932 unrelated individuals (sample 1) previously used for gene-longevity studies. ${ }^{16,17}$ Of these, 724 originated from the 1905 cohort (93 years of age), 626 participants originated from LSADT (age 70-90 years) and 582 participants originated from MADT (age 47-68 years). For twins only one twin from each pair was included. We then extended the study by replicating the initial positive findings in an independent sample of 541 individuals available from the 1905 survey (sample 2), which is the survey with the most events (highest mortality).

All participants were followed from the date of inclusion (November, 1996-May, 1999) to the date of either death or January 8, 2004, whichever came first, resulting in a mean follow-up time of 5 years for survivors (range 4.6-7.2 years). Information on survival status was retrieved from the Danish Central Population Register, which is continuously updated.

Self-reported information with regard to health issues was available from all surveys. As a part of the original survey, the participants were asked whether they did now or had previously suffered from various diseases. Only when the diagnosis had been made by a physician was a positive answer accepted as valid.

\section{Genotyping}

DNA was isolated from cheek swabs or blood spots using the QIAamp DNA Mini Kit (Qiagen). The three polymorphisms were detected by a Taqman-based allelic discrimination assay using the following primers and probes: -107C/T-sense: 5'-gcccagctagctgcgga-3'; -107C/ T-antisense: $5^{\prime}$-atcgatgggcgcagaca- $3^{\prime} ;-107 \mathrm{C}$ : $5^{\prime}$-FAM-cgccccgccctccc- $3^{\prime} ;$ 107T: $5^{\prime}$-TET-cccgcccaccctcc-3'; 55M/Lsense: $\quad 5^{\prime}$-tttctgttctcttttctggcagaaa-3'; 55M/L-antisense: $5^{\prime}$-gaaaacactcacagagctaatgaaagc-3'; 55M: 5'-FAM-ccattaggcagtatctccatgtcttcagagcc-3'; 55L: 5'-TET-tccattaggcagtatctccaagtcttcagagcc-3'; 199Q/R-sense: $5^{\prime}$-ggacctgagcacttttatggca-3'; 192Q/R-antisense: 5'-gacaacatacgaccacgctaaacc-3'; 192Q: 5'-FAM-ttcttgaccctacttacaatcctgggagatgt-3'; 192R: 5'-TET-cttgaccctacttacgatcctgggagatgt-3'. All primers and probes were designed using Primer Express software (Applied Biosystems).

DNA was amplified in a total volume of $10 \mu \mathrm{l}$ containing $5 \mu \mathrm{l}$ Taqman Universal Master Mix (Applied Biosystems), $900 \mathrm{~nm}$ of each primer (DNA Technology), $200 \mathrm{~nm}$ of each probe (MWG Biotech) and $\sim 10 \mathrm{ng}$ template DNA. PCR was performed in the ABI Prism 7700 using the conditions recommended by the manufacturer (Applied Biosystems) and analyzed using the Sequence Detection System software.

\section{Statistical analysis}

Genotype distribution frequencies were compared with the $\chi^{2}$ test. Haplotype frequencies were estimated using an expectation maximization algorithm and distribution differences tested using CLUMP. ${ }^{18}$ Linkage disequilibrium coefficients were estimated according to the method described by Chakravarti et al. ${ }^{19}$

Mortality risk was estimated using Cox's proportionalhazard model, adjusting for age and sex. Statistic procedures were performed with the STATA 8.0 statistical program (Stata Corporation).

\section{Results}

Baseline characteristics and genotype frequencies for each of the three polymorphisms are summarized in Table 1. Genotype frequencies were not different between men and women and combined frequencies are thus reported. A cross-sectional analysis indicated that there was no difference in genotype distribution in the three cohorts. Dividing the entire population into groups based on 10year intervals revealed no difference in genotype distribution either (results not shown). The estimated haplotype frequencies using all three polymorphisms were not significantly different in any groups and examination of haplotypes for two polymorphisms at a time did not 
Table 1 Characteristics and PON1 genotype frequencies

\begin{tabular}{|c|c|c|c|c|}
\hline & $\begin{array}{c}\text { MADT } \\
N=582(\text { sample 1) }\end{array}$ & $\begin{array}{c}\text { LSADT } \\
N=626 \text { (sample 1) }\end{array}$ & $\begin{array}{c}1905 \text { cohort } \\
N=724 \text { (sample 1) }\end{array}$ & $\begin{array}{c}1905 \text { cohort } \\
N=541 \text { (sample 2) }\end{array}$ \\
\hline $\begin{array}{l}\text { Age }^{\mathrm{a}} \\
\text { Male/female } \\
\text { Events (deaths) }\end{array}$ & $\begin{array}{c}53.6 \pm 5.7 \\
272 / 310\end{array}$ & $\begin{array}{c}77.8 \pm 4.4 \\
309 / 317\end{array}$ & $\begin{array}{c}93 \pm 0 \\
282 / 442\end{array}$ & $\begin{array}{c}93 \pm 0 \\
111 / 430\end{array}$ \\
\hline $\begin{array}{l}\text { Male/female } \\
\text { PON1 } 107 \mathrm{C} / T\end{array}$ & $10 / 6$ & $135 / 120$ & $232 / 319$ & $85 / 319$ \\
\hline $\begin{array}{l}\text { CC } \\
\text { CT } \\
\text { TT } \\
\text { PON1 } 55 \mathrm{M} / \mathrm{L}\end{array}$ & $\begin{array}{l}175(30.1 \%) \\
250(42.9 \%) \\
157(27.0 \%)\end{array}$ & $\begin{array}{l}173(27.6 \%) \\
298(47.6 \%) \\
155(24.8 \%)\end{array}$ & $\begin{array}{l}192(26.5 \%) \\
347(47.9 \%) \\
185(25.6 \%)\end{array}$ & \\
\hline $\begin{array}{l}\text { MM } \\
\text { ML } \\
\text { LL } \\
\text { PON1 } 192 Q / R\end{array}$ & $\begin{array}{c}74(12.7 \%) \\
265(45.5 \%) \\
243(41.8 \%)\end{array}$ & $\begin{array}{c}87(13.9 \%) \\
304(48.6 \%) \\
235(37.5 \%)\end{array}$ & $\begin{array}{c}96(13.3 \%) \\
334(46.1 \%) \\
294(40.6 \%)\end{array}$ & $\begin{array}{c}69(13.3 \%) \\
242(46.5 \%) \\
209(40.2 \%)^{b}\end{array}$ \\
\hline $\begin{array}{l}\text { QQ } \\
\text { QR } \\
\text { RR }\end{array}$ & $\begin{array}{c}307(52.8 \%) \\
230(39.5 \%) \\
45(7.7 \%)\end{array}$ & $\begin{array}{c}352(56.2 \%) \\
225(36.0 \%) \\
49(7.8 \%)\end{array}$ & $\begin{array}{c}381(52.6 \%) \\
290(40.1 \%) \\
53(7.3 \%)\end{array}$ & $\begin{array}{c}284(52.5 \%) \\
215(39.7 \%) \\
42(7.8 \%)\end{array}$ \\
\hline
\end{tabular}

${ }^{\mathrm{a}}$ Mean \pm SD.

${ }^{\mathrm{b}} \mathrm{N}=520$

MADT $=$ Study of Middle-Aged Danish Twins; LSADT = Longitudinal study of Ageing Danish Twins; 1905 cohort = The Danish 1905 Cohort.

change this picture (results not shown). All three polymorphisms showed linkage disequilibrium to some extent with $\Delta=-0.43$ for $108 \mathrm{C} / \mathrm{T}$ and $55 \mathrm{M} / \mathrm{L}, \Delta=-0.23$ for $108 \mathrm{C} /$ $\mathrm{T}$ and $192 \mathrm{Q} / \mathrm{R}$ and $\Delta=0.43$ for $55 \mathrm{M} / \mathrm{L}$ and $192 \mathrm{Q} / \mathrm{R}$, respectively.

In the 5-year follow-up period, a total of 822 out of the initially 1932 included individuals died. The age-adjusted mortality risk according to the three PON1 polymorphisms is shown in Tables 2 and 3 for women and men, respectively. The data indicate that the PON1 192Q/R polymorphism may be an independent mortality risk factor and most pronounced in women, the estimated hazard rate for RR homozygotic women being 1.38 (95\% CI: 1.01-1.87, $P=0.04)$ using the QQ genotype as the reference group, while the corresponding number for men was $1.14(P=0.55)$.

To confirm the results for this polymorphism in a new sample, we genotyped a further 541 individuals who were available from the 1905 cohort. A total of 404 out of these 541 individuals died during follow-up. The estimated hazard rate for RR homozygotic women in the second sample was $1.38(P=0.09)$, while effect was less significant in men (hazard rate $=1.31, P=0.66$ ). Looking at the combined group of samples 1 and 2 , the hazard rate was $1.38(P=0.008)$ for women and 1.20 for men $(P=0.43)$.

Adjusting for other available covariates, such as smoking status, BMI, hypertension, heart failure and self-rated health did not change the magnitude of the hazard rate estimates; hence, these are not included in the final analysis.

The increased mortality risk seen for 192RR homozygotic women was evident in all age groups above 70 years (only six women below 70 years of age died during the follow-up
Table 2 Mortality risk according to PON1 genotypes in a 5-year follow-up ${ }^{a}$ in women

\begin{tabular}{|c|c|c|c|}
\hline & Hazard rate & $95 \% \mathrm{Cl}$ & P-value \\
\hline \multicolumn{4}{|c|}{ PON1 genotypes } \\
\hline \multicolumn{4}{|c|}{ Sample $1(N=1069)$} \\
\hline$-107 C C$ & 1 & & \\
\hline$-107 \mathrm{CT}$ & 0.99 & $0.79-1.23$ & 0.90 \\
\hline$-107 T \mathrm{~T}$ & 1.08 & $0.83-1.40$ & 0.56 \\
\hline 55MM & 1669 & & \\
\hline $55 \mathrm{ML}$ & 0.69 & $0.52-0.90$ & 0.007 \\
\hline 55LL & 0.83 & $0.63-1.09$ & 0.17 \\
\hline 192QQ & 1 & & \\
\hline 192QR & 0.86 & $0.70-1.05$ & 0.13 \\
\hline 192RR & 1.38 & $1.01-1.87$ & 0.04 \\
\hline \multicolumn{4}{|c|}{ Sample $2(N=430)$} \\
\hline 192QQ & 1 & & \\
\hline 192QR & 1.05 & $0.83-1.33$ & 0.69 \\
\hline 192RR & 1.38 & $0.95-2.00$ & 0.09 \\
\hline $55 \mathrm{MM}$ & 1 & & \\
\hline $55 \mathrm{ML}$ & 1.17 & $0.82-1.69$ & 0.37 \\
\hline 55LL & 1.38 & $0.96-1.99$ & 0.08 \\
\hline \multicolumn{4}{|c|}{ Sample $1+2(N=1499)$} \\
\hline 192QQ & 1 & & \\
\hline 192QR & 0.93 & $0.80-1.09$ & 0.37 \\
\hline 192RR & 1.38 & $1.09-1.75$ & 0.008 \\
\hline
\end{tabular}

${ }^{a}$ Mean follow-up time for survivors was 5.1 years (range $4.7-7.1$ years).

${ }^{\mathrm{b}}$ Age-adjusted mortality risk using the genotypes $-107 \mathrm{CC}, 55 \mathrm{MM}$ and 192QQ as reference group, respectively.

period). We furthermore observed a significantly decreased mortality risk in $55 \mathrm{M} / \mathrm{L}$ heterozygotic women only (hazard rate $0.69, P=0.007$ ), and a non-significant trend towards a decreased risk in 55LL homozygotes (hazard rate 0.83, 
Table 3 Mortality risk according to PON1 genotypes in a 5-year follow-up ${ }^{a}$ in men

\begin{tabular}{llll}
\hline \multicolumn{5}{c}{ Hazard rate $^{\mathrm{b}}$} & $95 \% \mathrm{Cl}$ & P-value \\
\hline PON1 genotypes & & & \\
Sample 1 $(\mathrm{N}=863)$ & & & \\
$-107 \mathrm{CC}$ & 1 & & \\
$-107 \mathrm{CT}$ & 1.03 & $0.81-1.32$ & 0.80 \\
-107TT & 1.15 & $0.87-1.51$ & 0.33 \\
55MM & 1 & & \\
55ML & 0.99 & $0.72-1.35$ & 0.93 \\
55LL & 0.98 & $0.71-1.35$ & 0.88 \\
192QQ & 1 & & \\
192QR & 1.01 & $0.82-1.24$ & 0.94 \\
192RR & 1.14 & $0.74-1.78$ & 0.55
\end{tabular}

Sample $2(N=111)$

$\begin{array}{ll}\text { 192QQ } & 1 \\ \text { 192QR } & 0.98 \\ \text { 192RR } & 1.31 \\ 55 \mathrm{MM} & 1 \\ 55 \mathrm{ML} & 1.14 \\ 55 \mathrm{LL} & 0.86\end{array}$

$0.64-1.52$

$0.40-4.23$

0.94

31

1.14

$55 \mathrm{LL}$

$0.53-2.45$

$0.41-1.93$

0.66

0.74

0.76

Sample $1+2(\mathrm{~N}=974)$

192QQ 1

$192 Q R \quad 0.99$

192RR

1.20

$0.83-1.21$

$0.78-1.78$

0.98

0.43

${ }^{a}$ Mean follow-up time for survivors was 5 years (range 4.6-7.2 years).

${ }^{\mathrm{b}}$ Age-adjusted mortality risk using the genotypes $-107 \mathrm{CC}, 55 \mathrm{MM}$ and 192QQ as reference group, respectively.

$P=0.17$ ) compared to 55MM homozygotes. However, these results were not replicated in sample 2 .

Table 4 provides the genotype distribution for PON 192Q/R according to ischemic heart disease (angina and/or myocardial infarction). There is a nonsignificant increased proportion of RR homozygotes in women suffering from ischemic heart disease $(11.8 \%)$ compared to both men $(6.2 \%)$ and women $(8.4 \%)$ with no experience of ischemic heart disease.

\section{Discussion}

Although reports have been contradictory, several studies have suggested an association between PON1 polymorphisms and susceptibility to cardiovascular disease. As cardiovascular disease is one of the leading causes of death in middle-aged and elderly people, an impact of such polymorphisms on mortality could be expected.

In this study, we investigated the relation of three PON1 polymorphisms, $-107 \mathrm{C} / \mathrm{T}, 55 \mathrm{M} / \mathrm{L}$ and $192 \mathrm{Q} / \mathrm{R}$, with survival in a large sample of Danish individuals aged $47-3$ years, using both a cross-sectional and a longitudinal study design. The cross-sectional study revealed no difference in genotype distribution in neither men nor women for either of the polymorphisms when comparing different age groups, nor was there any change in haplotype distribution
Table 4 PON192Q/R genotypes according to self-reported ischemic heart disease $\mathrm{a}^{\mathrm{a}}$

\begin{tabular}{lrrr}
\hline & $192 Q Q$ & $192 Q R$ & \multicolumn{1}{c}{$192 \mathrm{RR}$} \\
\hline $\begin{array}{l}\text { Women } \\
\text { +Disease }\end{array}$ & $90(50.6 \%)$ & $67(37.6 \%)$ & $21(11.8 \%)$ \\
-Disease & $723(54.5 \%)$ & $492(37.1 \%)$ & $111(8.4 \%)$ \\
& & & \\
Men & & & \\
+Disease & $60(56.6 \%)$ & $40(37.7 \%)$ & $6(5.7 \%)$ \\
-Disease & $453(52.1 \%)$ & $363(41.7 \%)$ & $54(6.2 \%)$ \\
\hline
\end{tabular}

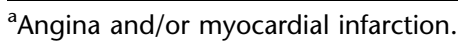

with age. The increased frequency of 55M/192R carriers with age reported by Bonafé et $a l^{11}$ could thus not be replicated here.

However, the longitudinal study, which could be expected to be more statistically powerful, revealed an increased mortality among women homozygous for 192RR, while the effect was smaller in men. Thus, in a 5year follow-up period, we found that 192RR homozygotic women had a mortality risk of $1.38(P=0.04)$ compared to individuals with the QQ genotype. Although not formally statistically significant, replication in a new sample (sample 2) confirmed this result (hazard rate: 1.38, $P=0.09$ ). Combining samples 1 and 2 did not change the risk estimate, but increased the statistical significance to $P=0.008$, thereby adding further support to the moderate but significant effect of this genotype on mortality risk in women.

We did not obtain information about the cause of death in this study, as the Danish Registry of Causes of Death is updated to year 2000 only, making it insufficient for the main part of the 5-year follow-up time used in this study. Hence, we cannot directly ascribe the reported poorer survival chance of 192RR homozygotic women to an increased susceptibility to CHD and death. Instead, we used self-reported data on ischemic heart disease (angina and/or acute myocardial infarction) in order to examine whether the increased mortality seen in 192RR women may possibly be caused by an increased susceptibility to development of CHD. We found a slightly higher proportion of RR homozygotes in the disease group compared to the nondisease group (11.8 vs 8.4\%) in women. However, this did not reach statistical significance. This observation is in line with the results of the recent meta-analysis conducted by Wheeler et $a l^{9}$ showing that PON 192Q/R was only weakly associated with CHD susceptibility.

As our data indicate an effect in the mortality pattern of RR homozygotes in women, this implies that PON1 192RR homozygosity, rather than being a susceptibility factor for CHD, is associated with CHD severity and survival after CHD. If PON1 192RR homozygosity is associated with higher mortality immediately after myocardial infarction, 
the association with CHD in case-control studies will be weaker than the association with survival. In support of this hypothesis, Chen et $a l^{20}$ recently reported an association between the PON1 192R allele and coronary artery disease severity in white women, when evaluating disease severity in terms of the number of diseased vessels.

Contrary to our findings, Heijmans et $a l^{10}$ found no significant effect of the Q192R and M55L polymorphisms on either cardiovascular or all caused mortality risks in 666 subjects age $85+$. This apparent discrepancy may be caused by the fact that our study population has a much larger age range (47-93 years), as well as the larger sample size in our study, which may be necessary to detect a moderately increased mortality risk as the one reported in the present study.

We furthermore observed a significantly decreased mortality risk in $55 \mathrm{M} / \mathrm{L}$ heterozygotes compared to $55 \mathrm{MM}$ homozygotes in women. The finding of a decreased risk for heterozygotes only is not compatible with either a recessive or a dominant effect of the 55L allele. Furthermore, this result was not replicated in the second sample. As we find some degree of pair-wise allelic association among all three polymorphisms $(\Delta=0.23-0.42)$, these deviating results for PON55 may merely reflect the partial linkage disequilibrium with PON192.

In conclusion, we found no substantial impact of PON1 polymorphisms or haplotypes on longevity, when comparing elderly Danish subjects to younger individuals in neither men nor women. However, the follow-up analysis suggested that, in particular, women homozygous for PON1 192RR may have an increased mortality risk in the second half of life.

\section{Acknowledgements}

We thank Shuxia Li for technical assistance. This work was supported by research grants from the National Institute on Aging (Grant NIAPO1-AG08761), the Danish Medical Research Council and the GENOMEUTWIN Project (European Union Contract No. QLG2-CT2002-01254).

\section{References}

1 Watson AD, Berliner JA, Hama SY et al: Protective effect of high density lipoprotein associated paraoxonase. Inhibition of the biological activity of minimally oxidized low density lipoprotein. I Clin Invest 1995; 96: 2882-2891.

2 Mackness MI, Arrol S, Durrington PN: Paraoxonase prevents accumulation of lipoperoxides in low-density lipoprotein. FEBS Lett 1991; 286: 152-154.

3 Mackness MI, Mackness B, Durrington PN, Connelly PW, Hegele RA: Paraoxonase: biochemistry, genetics and relationship to plasma lipoproteins. Curr Opin Lipidol 1996; 7: 69-76.
4 Heinecke JW, Lusis AJ: Paraoxonase-gene polymorphisms associated with coronary heart disease: support for the oxidative damage hypothesis? Am J Hum Genet 1998; 62: 20-24.

5 Adkins S, Gan KN, Mody M, La Du BN: Molecular basis for the polymorphic forms of human serum paraoxonase/arylesterase: glutamine or arginine at position 191, for the respective A or B allozymes. Am J Hum Genet 1993; 52: 598-608.

6 Leviev I, James RW: Promoter polymorphisms of human paraoxonase PON1 gene and serum paraoxonase activities and concentrations. Arterioscler Thromb Vasc Biol 2000; 20: 516-521.

7 Mackness B, Mackness MI, Arrol S, Turkie W, Durrington PN: Effect of the human serum paraoxonase 55 and 192 genetic polymorphisms on the protection by high density lipoprotein against low density lipoprotein oxidative modification. FEBS Lett 1998; 423: 57-60.

8 Deakin S, Leviev I, Brulhart-Meynet MC, James RW: Paraoxonase1 promoter haplotypes and serum paraoxonase: a predominant role for polymorphic position -107, implicating the Sp1 transcription factor. Biochem J 2003; 372 (Part 2): 643-649.

9 Wheeler JG, Keavney BD, Watkins PH, Collins PR, Danesh PJ: Four paraoxonase gene polymorphisms in 11212 cases of coronary heart disease and 12786 controls: meta-analysis of 43 studies. Lancet 2004; 363: 689-695.

10 Heijmans BT, Westendorp RG, Lagaay AM et al: Common paraoxonase gene variants, mortality risk and fatal cardiovascular events in elderly subjects. Atherosclerosis 2000; 149: $91-97$.

11 Bonafe M, Marchegiani F, Cardelli M et al: Genetic analysis of paraoxonase (PON1) locus reveals an increased frequency of Arg192 allele in centenarians. Eur J Hum Genet 2002; 10: 292-296.

12 Nybo H, Gaist D, Jeune B et al: The Danish 1905 cohort: a genetic-epidemiological nationwide survey. J Aging Health 2001; 13: $32-46$.

13 Christensen K, Gaist D, Vaupel JW, McGue M: Genetic contribution to rate of change in functional abilities among Danish twins aged 75 years or more. Am J Epidemiol 2002; 155: $132-139$.

14 Skytthe A, Kyvik K, Holm NV, Vaupel JW, Christensen K: The Danish Twin Registry: 127 birth cohorts of twins. Twin Res 2002; 5: 352-357.

15 Gaist D, Bathum L, Skytthe A et al: Strength and anthropometric measures in identical and fraternal twins: no evidence of masculinization of females with male co-twins. Epidemiology 2000; 11: 340-343.

16 Bathum L, Christiansen L, Nybo H et al: Association of mutations in the hemochromatosis gene with shorter life expectancy. Arch Intern Med 2001; 161: 2441-2444.

17 Christiansen L, Bathum L, Andersen-Ranberg K, Jeune B, Christensen K: Modest implication of interleukin 6 promoter polymorphisms in longevity. Mech Ageing Dev 2004; 125: $391-$ 395.

18 Sham PC, Curtis D: Monte Carlo tests for associations between disease and alleles at highly polymorphic loci. Ann Hum Genet 1995; 59 (Part 1): 97-105.

19 Chakravarti A, Buetow KH, Antonarakis SE et al: Nonuniform recombination within the human beta-globin gene cluster. Am J Hum Genet 1984; 36: 1239-1258.

20 Chen Q, Reis SE, Kammerer CM et al: Association between the severity of angiographic coronary artery disease and paraoxonase gene polymorphisms in the National Heart, Lung, and Blood Institute-sponsored Women's Ischemia Syndrome Evaluation (WISE) study. Am I Hum Genet 2003; 72: 13-22. 